\title{
LOCAL RIGIDITY AND GROUP COHOMOLOGY I: STOWE'S THEOREM FOR BANACH MANIFOLDS
}

\author{
VICTOR BRUNSDEN
}

\begin{abstract}
Stowe's Theorem on the stability of the fixed points of a $C^{2}$ action of a finitely generated group $\Gamma$ is generalised to $C^{\mathbf{1}}$ actions of such groups on Banach manifolds. The result is then used to prove that if $\phi$ is a $C^{r}$ action on a smooth, closed, manifold $M$ satisfying $H^{1}\left(\Gamma, D^{r-1}(M)\right)=0$, then $\phi$ is locally rigid. Here, $r \geqslant 2$ and $D^{k}(M)$ is the space of $C^{k}$ tangent vector fields on $M$. This generalises a local rigidity result of Weil for representations of a finitelv generated group $\Gamma$ in a Lie group.
\end{abstract}

\section{INTRODUCTION}

Let $\Gamma$ be a finitely generated group. Given a topological group $G$, the set of representations of $\Gamma$ in $G$ will be denoted by $R(\Gamma, G)$. This can be given the structure of a topological space by taking the topology to be the compact-open topology. The compact-open topology may also be shown to be the topology of uniform convergence on finite sets of generators of $\Gamma$. Given $\phi \in R(\Gamma, G)$, it is natural to try to determine the local structure of $R(\Gamma, G)$ in a neighbourhood of $\phi$. For the case of $G$ a Lie group with Lie algebra $\mathfrak{g}$, a classical result of Weil [14] states that if $H^{1}\left(\Gamma, \mathfrak{g}_{a d \phi}\right)=0$, then all representations $\psi$ of $\Gamma$ in a sufficiently small neighbourhood of $\phi$ in $R(\Gamma, G)$ are conjugate to $\phi$ via an element of $G$. The subscript $a d \phi$ refers to the action of $\Gamma$ on $\mathfrak{g}$ via the composition of $\phi$ with the adjoint action ad of $G$ on $\mathfrak{g}$. A representation $\phi$ of $\Gamma$ with this property is said to be locally rigid. The first cohomology group $H^{1}\left(\Gamma, \mathfrak{g}_{a d \phi}\right)$ may be computed using techniques such as those developed by Matsushima and Murakami [9].

Recently, there has been interest in extending this body of theory to a broader class of topological groups. Of interest is the diffeomorphism group $D_{i f f}(M)$ of a smooth closed manifold $M$. The analogue of the Lie algebra for $\operatorname{Diff}^{\infty}(M)$ is $D^{\infty}(M)$, the

\section{Received 7th September, 1998}

This paper is an extension of the author's $\mathrm{Ph}$.D dissertation at Binghamton University (formerly SUNY Binghamton). I would like to thank the faculty in the Department of Mathematical Sciences for their support. Most especially, I would like to thank my advisor, Tom Farrell for his very generous help and encouragement. I would also like to thank Nantian Qian for pointing out an error in a previous version of this paper.

Copyright Clearance Centre, Inc. Serial-fee code: 0004-9729/99 $\$ A 2.00+0.00$. 
space of $C^{\infty}$ tangent vector fields on $M$ and the analogue of $a d \phi$ is $\phi_{*}$. A representation of $\Gamma$ satisfying $H^{1}\left(\Gamma, D^{\infty}(M)_{\phi_{*}}\right)=0$ is said to be infinitesimally rigid. Zimmer [15] conjectured that, as for Lie groups, infintesimal rigidity implies local rigidity. The aim of this paper is to provide a partial answer in the affirmative to this.

Rather than proceeding directly, the approach presented here follows Fleming's observation [7] that Weil's original result may be deduced from a result of Stowe [13]. Stowe's theorem says that if $\phi \in R\left(\Gamma, D_{i f f}^{2}(M)\right)$ fixes a point $p \in M$, (here $M$ is assumed to be a smooth, finite dimensional manifold satisfying $\left.H^{1}\left(\Gamma, T_{p} M_{d \phi}\right)=0\right)$, then $p$ is stable; that is, for all neighbourhoods $\mathcal{U}$ of $p$ in $M$, there is a neighbourhood $\mathcal{W}$ of $\phi$ in $R\left(\Gamma, D_{i f f}{ }^{2}(M)\right)$ so that every $\psi \in \mathcal{W}$ has a fixed point in $\mathcal{U}$. The notation $d \phi$ refers to the linearisation of $\phi$ at the fixed point $p$. The fundamental theorem of this paper is a fixed point result generalising Stowe's Theorem. For notational convenience, let $\operatorname{Act}^{r}(\Gamma, M)$ be $R\left(\Gamma, \operatorname{Diff}^{r}(M)\right)$ where $1 \leqslant r \leqslant \infty$.

THEOREM 3.4. Let $\Gamma$ be a finitely generated group, $M$ a $C^{2}$ manifold modelled on a Banach space $V, \phi \in \operatorname{Act}^{1}(\Gamma, M)$ an action of $\Gamma$ on $M$ fixing a point $p \in M$ satisfing $H^{1}\left(\Gamma, V_{d \phi}\right)=0$, then, $p$ is stable.

Evidence for Zimmer's conjecture is provided by the following:

Corollary 4.3. Let $M$ be a smooth, closed manifold and $\phi \in \operatorname{Act}^{r}(\Gamma, M)$ a $C^{r}$ action of $\Gamma$ on $M$ satisfying $H^{1}\left(\Gamma, D^{r-1}(M)_{\phi^{*}}\right)=0$. Then, there is a neighbourhood $\mathcal{U}$ of $\phi$ in $\operatorname{Act}^{r}(\Gamma, M)$ so that any $\psi \in \mathcal{U}$ is conjugate to $\phi$ by an element $f \in \operatorname{Diff}^{r-1}(M)$, where $2 \leqslant r<\infty$.

Section 1 presents the basics needed for our discussion of Banach manifolds. While our manifolds are in general nothing more exotic than Banach manifolds, Fréchet spaces and other locally convex spaces do make an appearance and the needed results and notational conventions are given in 1.1. Section 1.2 presents the material that we shall need to define the topologies on various spaces of mappings from a Banach manifold to itself. Much of this is patterned after treatments of Eells [5] or Lang [8]. Cohomology is the subject of Section 2. More specifically, when the coefficient module $V$ carries the structure of a locally convex space a presentation of $\Gamma$ induces a topology on the set of cocycles associated to a Gruenberg resolution. The properties of this topology and the quotient topology inherited by the cohomology groups is investigated in Section 2.2. The main result is Theorem 2.5; for the coefficient module a Fréchet space and $\Gamma$ finitely generated, the (twisted) cohomology groups with coefficients are Hausdorff in dimensions $\geqslant 2$. This result relies on the vanishing of the higher dimensional cohomolgy for a free group. This is then used to derive the approximation result, Theorem 2.7. The idea is to approximate a cochain in a cochain complex of Fréchet spaces by a cocycle. This is done by examining its image under the coboundary map. The required 
approximating cocycle may then be constructed by identifying this image with the image of an element of the quotient of the space of cochains modulo cocycles under the natural injection. This leads to useful information only if the range of the coboundary map is closed. This approximation result is used in Section 3 to give a priori estimates for constructing cocycles from an action "close" to the original action. The main result, Theorem 3.4 then follows. The proof is constructive and differs from Stowe's even in the finite dimensional case. The theorem is then applied in Section 4 to derive the analogue of Weil's theorem for $\operatorname{Diff}^{r}(M)$ ( $r$ finite) and so confirm Zimmer's conjecture in this case.

\section{PRemmanianes}

Banach Manifolds and Topological Vector Spaces. All vector spaces are assumed real. Exceptions will be explicitly indicated. As always, for a locally convex topological vector space $V, V^{\prime}$ is its dual (= continuous, linear functionals), $V^{*}$ is $V^{\prime}$ with the topology of uniform convergence on bounded subsets of $V$ and the bidual $V^{* *}$ is the space $V^{* \prime}$ equipped with the topology of uniform convergence on bounded subsets of $V^{*}$.

Sources for material on Banach manifolds are Omori [10], Ebin and Marsden [6], Eells [5] or Lang [8]. Let $V$ be a Banach space. A paracompact, Hausdorff space $M$ is a Banach manifold modelled on $V, r \geqslant 0$ if there is a collection of pairs (called charts) $(U, \phi)_{U \in \mathcal{U}}$ where $\mathcal{U}$ is an open covering of $M$ and $\phi: U \rightarrow V$ is a homeomorphism of $U$ to an open subset of $V . M$ is a $C^{r}$ Banach manifold if for all charts $(U, \phi)$ and $(V, \psi)$ with $U \cap V \neq \emptyset$, the mapping $\phi \circ \psi^{-1}: \psi(U \cap V) \rightarrow \phi(U \cap V)$ is a $C^{r}$ mapping. As in the finite dimensional case the tangent bundle $T M$ of a $C^{r}$ Banach manifold $M$ is defined to be the $C^{r-1}$ Banach manifold modelled on the Banach space $V \times V$ with charts $(U \times V, \phi \times d \phi)_{U \in \mathcal{U}}$. The notions of a $C^{r}$ mapping between such spaces, its derivative and $k$-jet are as in the finite dimensional case and may be found in the previously mentioned references as well as in Deimling [4].

With the above definitions of a Banach manifold, it is a classical result that the group $\operatorname{Diff}^{r}(M)$ of $C^{r}$ diffeomorphisms of a smooth, closed manifold $M$, is itself a $C^{\infty}$ Banach manifold with its model Banach space being the space $D^{r}(M)$ of $C^{r}$ tangent vector fields to $M$ equipped with the topology of uniform convergence of derivatives of order $\leqslant r$ on compact subsets of $M$. The proof may be found in Ebin and Marsden [6], Omori [10], Eells [5] or Abraham [1]. With multiplication in $D_{i f f}{ }^{(M)}$ given by composition, $\operatorname{Diff}^{r}(M)$ becomes a topological group that is also a Banach manifold (not however, a Banach Lie group as right multiplication is not smooth). Another example of an infinite dimensional topological group having the structure of a Banach manifold is $C^{r}(M, G)$, the space of $C^{r}$ maps from a smooth, closed manifold $M$ to a Lie group 
$G$. Again the topology is that of uniform convergence of all derivatives of order $\leqslant r$ on compact subsets of $M$ and with this topology, it is a Banach Lie group.

The SPACE $R(\Gamma, G)$. Let $G$ be a topological group and $\Gamma$ a finitely generated group. Fix a presentation

$$
1 \longrightarrow R \longrightarrow F \longrightarrow \Gamma \longrightarrow 1
$$

of $\Gamma$, where $F$ is free on a finite subset $S$ of $\Gamma$ and $R$ is the kernel of the epimorphism mapping $F$ to $\Gamma$. Let $S$ consist of the elements $\gamma_{1}, \ldots, \gamma_{n}$ and $R$ be freely generated (see Robinson [12]) by $r_{1}, r_{2}, r_{3}, \ldots$ where $r_{i} \in F$ for $i=1, \ldots, n$. If $\psi: \Gamma \rightarrow G$ is a homomorphism, then clearly $\psi$ need only be specified on generators. In particular, since $\psi$ is a homomorphism,

$$
\psi\left(r\left(\gamma_{1}, \ldots, \gamma_{n}\right)\right)=r\left(\psi\left(\gamma_{1}\right), \ldots, \psi\left(\gamma_{n}\right)\right)
$$

for all $r \in R$. Suppose that $\psi\left(\gamma_{i}\right)=g_{i}$ for $i=1, \ldots, n$, then

$$
r\left(g_{1}, \ldots, g_{n}\right)=1_{G}
$$

for all $r \in R$. Conversely, given $g_{1}, \ldots, g_{n} \in G^{n}$ satisfying

$$
r\left(g_{1}, \ldots, g_{n}\right)=1_{G}
$$

for all $r \in R$, assigning $\psi\left(\gamma_{i}\right)=g_{i}$ for $\mathrm{i}=1, \ldots, n$ defines a homomorphism $\psi: \Gamma \rightarrow G$. In this fashion, for a presentation of $\Gamma$ as described above, the $\operatorname{set} \operatorname{Hom}(\Gamma, G)$, of homomorphisms from $\Gamma$ to $G$ may be regarded as the subset

$$
\left\{\left(g_{1}, \ldots, g_{n}\right) \in G^{n} \mid r\left(g_{1}, \ldots, g_{n}\right)=1_{G} \text { for all } r \in R\right\}
$$

of $G^{n}$. $G^{n}$ with the product topology thus induces a topology on $\operatorname{Hom}(\Gamma, G)$. It is straightforward to check that this topology is independent of the presentation and coincides with the compact-open topology on $\operatorname{Hom}(\Gamma, G)$ when $\Gamma$ is given the discrete topology. We define $R(\Gamma, G)$ to be $\operatorname{Hom}(\Gamma, G)$ equipped with this topology. If $M$ is a smooth closed manifold, let $\operatorname{Act}^{r}(\Gamma, M)$ be the space $R\left(\Gamma, \operatorname{Diff}^{r}(M)\right)$, where $\operatorname{Diff}^{r}(M)$ is given the topology of uniform convergence of derivatives of order $\leqslant r$ on compact subsets of $M$.

Finally, we define the Fox derivative. Given a presentation of $\Gamma$,

$$
1 \longrightarrow R \longrightarrow F \longrightarrow \Gamma \longrightarrow 1
$$

where $F$ is free on $S \subset \Gamma$ as above, $\psi \in \operatorname{Hom}(\Gamma, \operatorname{Aut}(A))$ and $\left(a_{1}, \ldots\right) \in A^{|S|}$, the Fox derivative of $w \in F$ is defined as follows. Composition of $\psi$ with the quotient 
homomorphism $F \longrightarrow \Gamma$ gives a homomorphism which will also be denoted by $\phi$. Let $S=\left\{\gamma_{i}\right\}_{i \in \mathcal{I}}$, then given $\left\{a_{i}\right\}_{i \in \mathcal{I}}$ where $a_{i} \in A$ there is $\hat{\phi} \in \operatorname{Hom}(F, A f f(A))$ with $\widehat{\phi}\left(\gamma_{i}\right)(a)=\phi\left(\gamma_{i}\right)(a)+a_{i}$ for all $i \in \mathcal{I}$. The Fox derivative of $w \in F$, written $d w_{\phi}\left(a_{1}, \ldots\right)$, is then defined to be $\widehat{\phi}\left(w\left(\gamma_{1}, \ldots\right)\right)(0)$. The Fox derivative satisfies $d\left(w_{1} w_{2}\right)_{\phi}\left(a_{1}, \ldots\right)=d\left(w_{1}\right)_{\phi}\left(a_{1}, \ldots\right)+\phi\left(w_{1}\left(\gamma_{1}, \ldots\right)\right)\left(d\left(w_{2}\right)_{\phi}\left(a_{1}, \ldots\right)\right)$ for all words $w_{1}, w_{2} \in F$.

\section{Cономоlogy}

FRÉCHET SPACES. The following results will be useful for dealing with the Fréchet spaces that occur in this paper. The first of these may be found in [2, Proposition 12

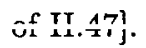

Proposition 2.1. Let $V$ and $W$ be two Fréchet spaces and $F$ a continuous linear mapping from $V$ onto $W$. Then there exists a section of $F$ that is continuous though not necessarily linear.

In the following, a normed Fréchet space is a Fréchet space equipped with a continuous norm \|\| . Note that the norm does not define the topology unless the space happens to be a Banach space. An example of such a space is the space of $C^{\infty}(M)$ real valued functions on a smooth, compact manifold $M$ with \|\| being the $C^{0}$ norm. With these conventions, Proposition 2.1 can be strengthened to

Proposition 2.2. Let $V$ be a normed Fréchet space with norm \|\| and $X$ a closed subspace. Assume that $Y=V / X$ with $F: V \rightarrow Y$ the quotient mapping is also a normed Fréchet space with norm \|\|$_{Y}$. Then for any $\eta>0$ there is a section $\sigma: Y \rightarrow V$ with

$$
\|\sigma(y)\| \leqslant(1+\eta)\|y\|_{Y}
$$

Proof: Let $\tau: Y \rightarrow V$ be the section of $F$ given by Proposition 2.1. For all $y \in Y$ there is a (non unique) $x \in X$ so that $\|y\|_{Y}+\eta>\|\tau(y)+x\|$. As $\tau$ is continuous, there is an open neighbourhood $W_{y}$ of $y$ so that $\left\|y^{\prime}\right\|_{Y}+\eta>\left\|r\left(y^{\prime}\right)+x\right\|$ for all $y^{\prime} \in W_{y}$. The collection $\left\{W_{y} \mid y \in Y\right\}$ is an open cover of $Y$. As $Y$ is paracompact, there is a locally finite subcover $\left\{U_{i}\right\}_{i \in I}$ and constant mappings $\left\{t_{i}: Y \rightarrow X\right\}_{i \in I}$ so that $\|y\|_{Y}+\eta>\left\|\tau(y)+t_{i}(y)\right\|$ for all $y \in U_{i}$. If $\chi_{i}$ is a partition of unity subordinate to the cover $\left\{U_{i}\right\}_{i \in I}$ then the section $\tau_{1}(y)=\tau(y)+\sum_{i \in I} \chi_{i}(y) t_{i}(y)$ satisfies $\left\|\tau_{1}(y)\right\|<\|y\|_{Y}+\eta$ for all $y \in Y$. Define $\sigma$ on $Y-\{0\}$ by

$$
\sigma(y)=\|y\|_{Y} \tau_{1}\left(\frac{y}{\|y\|_{Y}}\right)
$$

By construction it satisfies the desired inequality and is clearly continuous on $Y-\{0\}$. Extending $\sigma$ to all of $Y$ by $\sigma(0)=0$ completes the construction of $\sigma$. 
Chain Complexes. The cohomology groups occurring in this paper arise from (co)chain complexes built out of the coefficient group (always a Fréchet space in this context) and a projective $\mathbb{Z}[\Gamma]$ resolution of $\mathbb{Z}$. The cochain groups inherit a topology from the coefficients that depends on the resolution. Therefore the cohomology groups of such a complex inherit a topology as sub-quotients of the complex that is a priori dependent on the topology of the complex. For cohomology with coefficients in a Fréchet space, this will be shown to be independent of the resolution. Given a cochain complex $\left(C^{\bullet}, \partial^{\bullet}\right)$

$$
C^{0} \stackrel{\partial^{0}}{\longrightarrow} C^{1} \stackrel{\partial^{1}}{\longrightarrow} C^{2} \stackrel{\partial^{2}}{\longrightarrow} \ldots
$$

let $Z^{i}\left(C^{\bullet}\right)=\operatorname{ker}\left(\partial^{i}\right)$ denote the cocoycles in degree $i$ and $B^{i}\left(C^{\bullet}\right)=\operatorname{im}\left(\partial^{i-1}\right)$ the coboundaries in degree $i$. Usually, a cochain complex will simply be referred to as $C^{\bullet}$ with the coboundary operators $\partial^{\bullet}$ being understood. The complexes arise by applying the functor $\operatorname{Hom}_{\Gamma}(, V)$ to a projective $\mathbb{Z}[\Gamma]$ resolution

$$
\ldots \stackrel{\partial_{n+1}}{\longrightarrow} P_{n} \stackrel{\partial_{n}}{\longrightarrow} P_{n-1} \stackrel{\partial_{n-1}}{\longrightarrow} \ldots \stackrel{\partial_{1}}{\longrightarrow} P_{0} \stackrel{\partial_{0}}{\longrightarrow} \mathbb{Z} \stackrel{\partial}{\longrightarrow} 0
$$

of $\mathbb{Z}$. As this type of construction will occur repeatedly throughout this section, it will be worthwhile to record some of the topological properties inherited by the image of the functor $\operatorname{Hom}_{\Gamma}(, V)$ when $V$ is a Fréchet space in the following. For any (countable) ring $R$ together with a countably generated $R$ module $T$ and action of $R$ on a Fréchet space $V$ via continuous linear transformations, the Abelian group

$$
\operatorname{Hom}_{R}(T, V) \subset \prod_{t \in T} V
$$

may be given the topology of a Fréchet space by first giving $\prod_{t \in T} V$ the product topology (so making it a Fréchet space) and then considering $\operatorname{Hom}_{R}(T, V)$ as the subspace of $\prod_{t \in T} V$ defined by

$$
\operatorname{Hom}_{R}(T, V)=\left(\bigcap_{t, t^{\prime} \in T} L_{t, t^{\prime}}^{-1}(0)\right) \bigcap\left(\bigcap_{r \in R, t \in T} M_{r, t}^{-1}(0)\right)
$$

where $L_{t, t^{\prime}}(x)=x(t)+x\left(t^{\prime}\right)-x\left(t+t^{\prime}\right)$ and $M_{r, t}(x)=r . x(t)-x(r t)$ and function notation is used for the product $\prod_{t \in T} V$. Clearly, both $L_{t, t^{\prime}}$ and $M_{r, t}$ are continuous on $\prod_{t \in T} V$ and so $\operatorname{Hom}_{R}(T, V)$ is a closed subspace of $\prod_{t \in T} V$. Therefore, if $V$ is a Fréchet space, so is $\operatorname{Hom}_{R}(T, V)$ if $T$ is countable. Similarly, applying $\operatorname{Hom}_{R}(, V)$ to $f \in \operatorname{Hom}_{R}(S, T)$ produces a map of topological vector spaces that may be verified to be 
continuous when both $\operatorname{Hom}_{R}(T, V)$ and $\operatorname{Hom}_{R}(S, V)$ are given the topology outlined above. Moreover, if $T$ is free as an $R$-module on a set $X$, then as a Fréchet space

$$
\operatorname{Hom}_{R}(T, V)=\prod_{x \in X} V
$$

and if $S \subset T$ is a projective submodule (that is, $S$ is a direct summand of $T$ ), then it is straightforward to verify that $\operatorname{Hom}_{R}(S, V)$ is a direct summand of $\operatorname{Hom}_{R}(T, V)$. Furthermore, if $f \in \operatorname{Hom}_{R}(T, S)$ is a surjective $R$-homomorphism then the induced map of Fréchet spaces $f^{*}: \operatorname{Hom}_{R}(S, V) \rightarrow \operatorname{Hom}_{R}(T, V)$ is continuous. Moreover, since

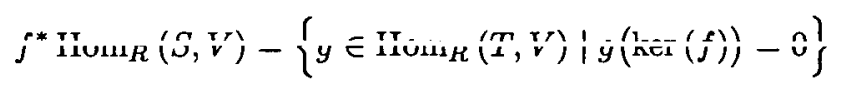

this exhibits $\operatorname{im}\left(f^{*}\right)$ as a closed subspace of $\operatorname{Hom}_{R}(T, V)$. As these constructions will be used repeatedly in this section, here is a summary of them in the following lemma.

Lemma 2.3. If $V$ is a Fréchet space, $R$ a (countable) ring acting via continuous linear transformations on $V$, then

(1) $\operatorname{Hom}_{R}(, V)$ takes countable $R$-modules to Fréchet spaces and

(2) $R$-module homomorphisms to continuous linear maps of Fréchet spaces, and

(3) projective, countably generated $R$-modules to direct summands of countable direct products of $V$.

(4) If $f \in \operatorname{Hom}_{R}(T, S)$ is a surjective $R$-homomorphsim, $f^{*} \operatorname{Hom}_{R}(S, V)$ is a closed subspace of $\operatorname{Hom}_{R}(T, V)$.

Applying Lemma 2.3 to the ring $\mathbb{Z}[\Gamma]$ and a projective $\mathbb{Z}[\Gamma]$ resolution of $\mathbb{Z}$ gives a cochain complex $\left(C^{\bullet}, \partial^{\bullet}\right)$

$$
0 \stackrel{\partial}{\longrightarrow} \operatorname{Hom}_{\Gamma}(\mathbb{Z}, V) \stackrel{\partial^{0}}{\longrightarrow} \operatorname{Hom}_{\Gamma}\left(P_{0}, V\right) \stackrel{\partial^{1}}{\longrightarrow} \operatorname{Hom}_{\Gamma}\left(P_{1}, V\right) \stackrel{\partial^{2}}{\longrightarrow} \ldots
$$

where the modules in the complex are Fréchet spaces and the coboundary maps are continuous maps of Fréchet spaces. We say that a cochain complex $C^{\bullet}$ is a cochain complex of locally convex topological vector spaces if each $C^{i}$ is a locally convex, topological vector space and the coboundary maps $\partial^{i}: C^{i} \rightarrow C^{i+1}$ are continuous and is a Fréchet cochain complex if in addition each $C^{i}$ is a Fréchet space. Therefore, the above considerations imply that a resolution by countably generated projective modules gives rise to a Fréchet cochain complex if $V$ is itself a Fréchet space. The cohomology groups of such a complex will inherit a topology from the complex that a priori depends on the resolution. For the case of a complex whose cohomology is $\Gamma$ cohomology with Fréchet space coefficients, we shall show this to be independent of the resolution. 
LEMma 2.4. Let $C^{\bullet}$ and $D^{\bullet}$ be two cochain complexes of locally convex topological vector spaces, $f^{\bullet}: C^{\bullet} \rightarrow D^{\bullet}$ a (continuous) morphism and $i \in \mathbb{N}$. Then if $f_{*}^{i}: H^{i}\left(D^{\bullet}\right) \rightarrow H^{i}\left(C^{\bullet}\right)$ is bijective, the topology induced on $H^{i}\left(C^{\bullet}\right)$ by pulling back the topology on $H^{i}\left(D^{\bullet}\right)$ via $f_{*}^{\bullet}$ is coaser than the topology on $H^{i}\left(C^{\bullet}\right)$ coming from $C^{\bullet}$.

PRoOF: By definition, for any cohomology class $z+B^{i-1}\left(C^{\bullet}\right)$ in $H^{i}\left(C^{\bullet}\right)$

$$
f_{*}^{i}\left(z+B^{i-1}\left(C^{\bullet}\right)\right)=f^{i}(z)+B^{i-1}\left(D^{\bullet}\right)
$$

As $f^{\bullet}$ is continuous, the induced map on the quotient $Z^{i}\left(C^{\bullet}\right) / B^{i}\left(C^{\bullet}\right)$ is continuous and the result follows.

Given two projective $\mathbb{Z}[\Gamma]$ resolutions $P_{\bullet} \rightarrow \mathbb{Z}$ and $P_{\bullet}^{\prime} \rightarrow \mathbb{Z}$ of $\mathbb{Z}$, the induced homotopy equivalence of the resolutions $\varepsilon: P_{\bullet} \rightarrow P_{\bullet}^{\prime}$ and $\varepsilon^{\prime}: P_{\bullet}^{\prime} \rightarrow P_{\bullet}$ induce continuous maps of the cochain complexes $\varepsilon^{\bullet}: \operatorname{Hom}_{\Gamma}\left(P_{\bullet}^{\prime}, V\right) \rightarrow \operatorname{Hom}_{\Gamma}\left(P_{\bullet}, V\right)$ and $\varepsilon_{1 \bullet}: \operatorname{Hom}_{\Gamma}\left(P_{\bullet}, V\right) \rightarrow \operatorname{Hom}_{\Gamma}\left(P_{\bullet}^{\prime}, V\right)$ for any coefficient module $V$ carrying the structure of a locally convex topological vector space with a continuous $\Gamma$ action. Therefore, the previous Lemma implies that the topologies induced on $H^{\bullet}(\Gamma, V)$ from either resolution are identical. In this fashion, we may talk about the topology on $H^{\bullet}(\Gamma, V)$ being induced from the topology on $V$.

The previous considerations show that the cochain complex $\operatorname{Hom}_{\Gamma}\left(P_{\bullet}, V\right)$ with $P_{\bullet}$ countably generated and $V$ a Fréchet space, is a Fréchet chain complex. Moreover, if $\Gamma$ is $F P_{n}$ (that is, there is a projective $\mathbb{Z}[\Gamma]$ resolution $P_{0}$ of $\mathbb{Z}$ with $P_{i}$ finitely generated for all $i \leqslant n)$ then, if $V$ is a Banach space, so is $\operatorname{Hom}_{\Gamma}\left(P_{i}, V\right)$. Following Borel and Wallach [3], we say that a cochain complex of locally convex topological vector spaces $V^{\bullet}$ has Hausdorff cohomology in dimension $i$ if $H^{i}\left(V^{\bullet}\right)$ is Hausdorff and has Hausdorff cohomology if $H^{i}\left(V^{\bullet}\right)$ is Hausdorff for all $i$, equivalently if im $\left(\delta^{i}\right)$ is a closed subspace of $V^{i+1}$. Similarly, we shall say that $H^{i}(\Gamma, V)$ is Hausdorff if $\operatorname{Hom}_{\Gamma}\left(P_{\bullet}, V\right)$ has Hausdorff cohomology in dimension $i$ for at least one (hence any) projective $\mathbb{Z}[\Gamma]$ resolution $P_{\bullet}$ of $\mathbb{Z}$.

A Fréchet cochain complex computing the cohomology of a finitely generated group $\Gamma$ may obtained from the Gruenberg Resolution corresponding to a presentation

$$
1 \longrightarrow R \longrightarrow F \longrightarrow \Gamma \longrightarrow 1
$$

of $\Gamma$ where $F$ is a free group on a finite set of generators $S \subset \Gamma$. The Gruenberg resolution associates to such a presentation a free $\mathbb{Z}[\Gamma]$ resolution $P_{\bullet}$

$$
\ldots \stackrel{\partial_{n+1}}{\longrightarrow} P_{n} \stackrel{\partial_{n}}{\longrightarrow} P_{n-1} \stackrel{\partial_{n-1}}{\longrightarrow} \ldots \stackrel{\partial_{1}}{\longrightarrow} P_{0} \stackrel{\partial_{0}}{\longrightarrow} \mathbb{Z} \stackrel{\partial}{\longrightarrow} 0
$$


of $\mathbb{Z}$, where the $P_{i}$ s are countably generated if $\Gamma$ is. The modules $P_{n}$ are defined via

$$
\begin{aligned}
P_{2 n+1} & =I_{F} \bar{I}_{R}^{n} / I_{F} \bar{I}_{R}^{n+1} \\
P_{2 n} & =\bar{I}_{R}^{n} / \bar{I}_{R}^{n+1}
\end{aligned}
$$

where $I_{G}$ is the kernel of the $G$ map $\mathbb{Z}[G] \rightarrow \mathbb{Z}$ and $\bar{I}_{N}=\mathbb{Z}[G] I_{N}$ is the kernel of the homomorphism $\mathbb{Z}[G] \rightarrow \mathbb{Z}[G / N]$ for any normal subgroup $N$ of a group $G$. The coboundary maps are defined by $\partial^{n}(f)(x)=f\left(\partial_{n}(x)\right)$ for $f \in \operatorname{Hom}_{\Gamma}\left(P_{n}, V\right)$. A full treatment of the construction of these can be found in [12]. For our purposes, the important result on Hausdorff cohomology is:

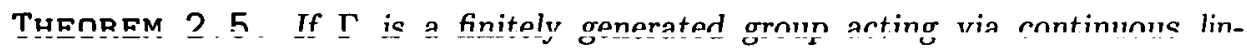
ear transformations on a Fréchet space $V$ then the Fréchet cochain complex $C^{\bullet}=$ $\operatorname{Hom}_{\Gamma}\left(P_{\bullet}, V\right)$ has Hausdorff cohomology in dimension $\geqslant 2$.

Proof: It suffices to show that $\partial^{\bullet}: \operatorname{Hom}_{\Gamma}\left(P_{\bullet}, V\right) \rightarrow \operatorname{Hom}_{\Gamma}\left(P_{\bullet+1}, V\right)$ is open, as then the subspace topology on $\operatorname{im}\left(\partial^{\bullet}\right)$ coincides with the quotient topology on $\operatorname{Hom}_{\Gamma}\left(P_{\bullet}, V\right) / \operatorname{ker}\left(\partial^{\bullet}\right)$. As the quotient is a Fréchet space, this shows that the image of $\partial^{\bullet}$ is closed and so $H^{\bullet}$ is Hausdorff. The idea of the proof is to map the complex $C^{\bullet}$ to another Fréchet cochain complex $\bar{C}^{\bullet}$ with vanishing cohomology for $\bullet>1$. With some care, $C^{\bullet}$ will be embedded as a closed subcomplex of $\bar{C}^{\bullet}$ and so $B^{\bullet}\left(\bar{C}^{\bullet}\right)=$ $\operatorname{im}\left(\bar{\partial}^{\bullet}\right)$ will be a closed subspace of $\operatorname{Hom}_{\Gamma}\left(P_{\bullet}, V\right)$ for $\bullet>1$. The particular complex chosen computes $H^{\bullet}(F, V)$ where $1 \longrightarrow R \longrightarrow F \longrightarrow \Gamma \longrightarrow 1$ is a presentation of $\Gamma$ and $V$ is given the structure of a $\mathbb{Z}[F]$ module via the natural homomorphism $F \longrightarrow \Gamma \longrightarrow G L(V)$ induced by the presentation and the action of $\Gamma$ on $V$.

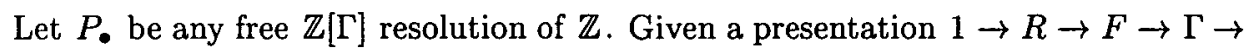
1 of $\Gamma$, construct a free $\mathbb{Z}[F]$ resolution $\bar{P}$. of $\mathbb{Z}$ with a surjective $\mathbb{Z}[F]$ homomorphism $\sigma_{\bullet}: \bar{P}_{\bullet} \rightarrow P_{\bullet}$ of resolutions as follows. For the initial segment

$$
P_{0} \longrightarrow \mathbb{Z} \longrightarrow 0,
$$

by regarding $P_{0}$ as a $\mathbb{Z}[F]$ module in the fashion described above, let $\bar{P}_{0}$ be a free $\mathbb{Z}[F]$ module mapping surjectively to $P_{0}$ via $\sigma_{0}$ and $\bar{\partial}_{0}=\partial_{0}\left(\sigma_{0}\right)$ be the composition of this homomorphism with $\partial_{0}$ so that

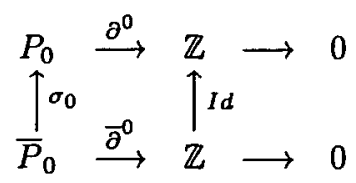

commutes. Proceeding by induction, assume that $\left(\bar{P}_{\bullet}, \bar{\partial}_{\bullet}\right)$ has been constructed for - $\leqslant n$. Let $\bar{Q}_{n+1}$ be a free $\mathbb{Z}[F]$ module with a surjective $\mathbb{Z}[F]$ homomorphism $\bar{s}_{n+1}$ 
to $P_{n+1}$ and $Q_{n+1}$ a free $\mathbb{Z}[F]$ module with a surjective homomorphism $d_{n+1}$ to $\operatorname{ker}\left(\bar{\partial}_{n}: \bar{P}_{n} \rightarrow \bar{P}_{n-1}\right)$. By the inductive hypothesis, $\sigma_{n}: \bar{P}_{n} \rightarrow P_{n}$ is surjective and is also a map of complexes, hence we may lift $\sigma_{n} \circ s_{n+1}$ to a homomorphism $r_{n+1}$ : $Q_{n+1} \rightarrow \bar{Q}_{n+1}$ and similarly define a lift $\bar{r}_{n+1}: \bar{Q}_{n+1} \rightarrow \operatorname{ker}\left(\bar{\partial}_{n}\right)$ of $s_{n+1}$ so that the diagrams

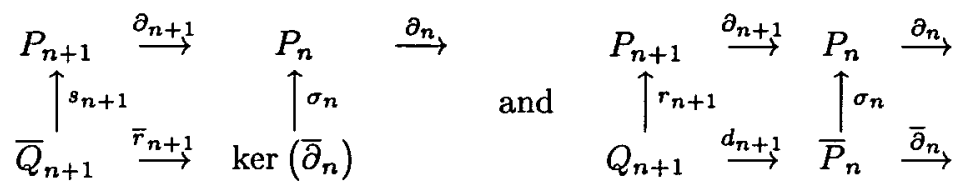

commute. Now define $\bar{P}_{n+1}=Q_{n+1} \oplus \bar{Q}_{n+1}, \sigma_{n+1}=r_{n+1}+s_{n+1}$ and $\bar{\partial}_{n+1}=$ $d_{n+1}+\bar{r}_{n+1}$. Clearly this completes the inductive step. Moreover, if $P_{\bullet}$ is countable, then clearly $\bar{P}$. may be chosen to be countable for all $\bullet \geqslant 0$.

Embed $\operatorname{Hom}_{\Gamma}\left(P_{0}, V\right)$ into $\operatorname{Hom}_{F}\left(P_{\bullet}\right)$ via the natural action $F$ on $P_{0}$ and $V$ coming from the quotient homomorphism $\pi: F \rightarrow \Gamma$. Let $\sigma^{\bullet}: \operatorname{Hom}_{F}\left(P_{\bullet}, V\right) \rightarrow$ $\operatorname{Hom}_{F}\left(\bar{P}_{\bullet}, V\right)$ be the composition of this identification with $\sigma_{\bullet}^{*}: \operatorname{Hom}_{F}\left(P_{\bullet}, V\right) \rightarrow$ $\operatorname{Hom}_{F}\left(\bar{P}_{\bullet}, V\right)$, which by construction is a morphism of Fréchet cochain complexes. The definition of $\bar{P}_{\bullet}$ and $\sigma_{\bullet}$ embeds $\sigma^{\bullet}\left(\operatorname{Hom}_{F}\left(P_{\bullet}, V\right)\right)$ as the closed subspace $\{f \in$ $\operatorname{Hom}_{F}\left(\bar{P}_{\bullet}, V\right) \mid f(p+r)=f(p)$ for all $\left.r \in \operatorname{ker}\left(\sigma_{\bullet}\right)\right\}$ of $\operatorname{Hom}_{F}\left(\bar{P}_{\bullet}, V\right)$.

By construction, the Fréchet cochain complex $\operatorname{Hom}_{F}\left(\bar{P}_{\bullet}, V\right)$ has cohomology groups $H^{\bullet}(F, V)$ which are 0 for $\bullet \geqslant 2$. Therefore the subspace topology on $\operatorname{im}\left(\bar{\partial}^{\bullet}\right)$ is identical to the quotient topology on $\vec{C}^{\bullet} / \operatorname{ker}\left(\bar{\partial}^{\bullet}\right)$ for $\bullet \geqslant 1$ where

$$
\bar{C}^{\bullet}=\operatorname{Hom}_{F}\left(\bar{P}_{\bullet}, V\right) \text {. }
$$

Now consider the diagram

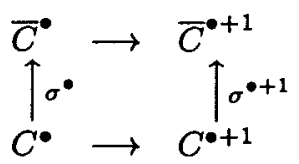

for $\bullet \geqslant 1$. Choosing the complex $\bar{P}$. to be countably generated for each $\bullet$ gives a Fréchet cochain complex. Choose a (continuous) section $d^{\bullet}: \operatorname{im}\left(\bar{\partial}^{\bullet}\right) \rightarrow \bar{C}^{\bullet}$ of $\bar{\partial}^{\bullet}$ with $d^{\bullet}(0)=0$. The section $d^{\bullet}$ gives rise to a homeomorphism of topological spaces (though not an isomorphism of Fréchet spaces)

$$
\left(I d-d^{\bullet} \bar{\partial}^{\bullet}\right) \oplus \bar{\partial}^{\bullet}: \bar{C}^{\bullet} \rightarrow \operatorname{ker}\left(\bar{\partial}^{\bullet}\right) \oplus \operatorname{im}\left(\bar{\partial}^{\bullet}\right)
$$

with inverse

$$
\iota+d^{\bullet}: \operatorname{ker}\left(\bar{\partial}^{\bullet}\right) \oplus \operatorname{im}\left(\bar{\partial}^{\bullet}\right) \rightarrow \bar{C}^{\bullet}
$$


where $\iota: \operatorname{ker}\left(\bar{\partial}^{\bullet}\right) \rightarrow \bar{C}^{\bullet}$ is the inclusion. However there are neighbourhoods $Y$ and $Z$ of 0 in $\operatorname{ker}\left(\bar{\partial}^{\bullet}\right)$ and $\operatorname{im}\left(\bar{\partial}^{\bullet}\right)$ respectively, so that

$$
\left(\sigma^{\bullet}\right)^{-1}\left(\left(\iota+d^{\bullet}\right)(Y \oplus Z)\right) \subset U .
$$

Clearly, $Z \subset \partial^{\bullet}(U)$ and so $\partial^{\bullet}(U)$ is open in $\operatorname{im}\left(\partial^{\bullet}\right)$ as required.

Lemma 2.6. Let $C^{\bullet}$ be a Fréchet cochain complex with Hausdorff cohomology in dimension 2. Then for any seminorm $p$ on $C^{i}$ there is a seminorm $q$ on $C^{1}$ so that

$$
\inf _{z \in Z^{1}} p(c+z) \leqslant q\left(\partial^{1}(c)\right)
$$

for all $c \in C^{1}$.

Proof: By assumption $B^{2}\left(C^{\bullet}\right) \leqslant C^{2}$ is a closed subspace and so is a Fréchet space. Hence, $\partial^{1}: C^{1} \rightarrow B^{2}\left(C^{\bullet}\right)$ is a continuous surjective linear map of Fréchet spaces and so by Bourbaki [2, Proposition IV 4.1.1], $B^{2}\left(C^{\bullet}\right)$ is isomorphic (as a Fréchet space) to $C^{1} / Z^{1}\left(C^{\bullet}\right)$. The topology on the quotient space $C^{1} / Z^{1}\left(C^{\bullet}\right)$ is given by seminorms of the form $\inf _{z \in Z^{1}} p(+z)$. The result now follows since any set of seminorms on $B^{2}\left(C^{\bullet}\right)$ giving the Fréchet space topology for $B^{2}\left(C^{\bullet}\right)$ is equivalent to the above set of seminorms on $B^{2}=C^{1} / Z^{1}\left(C^{\bullet}\right)$.

The low dimensional cohomology groups can be given the following interpretations. The 0 -th cohomology group may be identified as

$$
H^{0}(\Gamma, V)=\{v \in A \mid v=\varphi(\gamma) v \text { for all } \gamma \in \Gamma\} .
$$

The group of 1-cocycles may be interpreted to be

$$
Z^{1}(\Gamma, V)=\{z: \Gamma \rightarrow V \mid v \mapsto \varphi(\gamma) v+z(\gamma) \in \operatorname{Hom}(\Gamma, A f f(V))\}
$$

A function $z: \Gamma \rightarrow V$ satisfying this condition is called a crossed homomorphism. The subgroup consisting of the coboundaries may be interpreted as the set of all cocycles for which there is an $a \in A$ which is fixed by this affine action. That is, $\varphi(\gamma) a+z(\gamma)=a$ for all $\gamma \in \Gamma$. They are referred to as principal crossed homomorphisms. The first cohomology group with coefficients in $A$ is then the quotient of the group of crossed homomorphisms by the group of principal crossed homomorphisms.

For the Gruenberg resolution corresponding to the presentation $1 \longrightarrow R \rightarrow F \rightarrow$ $\Gamma \longrightarrow 1$, there is an exact sequence

$$
Z^{1}(F, A) \stackrel{\rho}{\longrightarrow} \operatorname{Hom}_{\Gamma}\left(R_{a b}, A\right) \longrightarrow H^{2}(\Gamma, A) \longrightarrow 0
$$

that will provide useful estimates for approximating cochains by cocycles [12, Theorem 11.4.9]. 
TheOREM 2.7. Let $V$ be a Fréchet space, $\psi \in R(\Gamma, V)$ with $1 \rightarrow R \rightarrow F \longrightarrow$ $\Gamma \longrightarrow 1$ a presentation of $\Gamma$ with $F$ finitely generated by $S \subset \Gamma$ and $R$ generated by $T=\left\{r_{1}, r_{2}, \ldots\right\} \subset F$. Let $\left\{p_{n}\right\}_{n \in \mathbb{N}}$ be an increasing set of seminorms defining the topology on $V$, that is, $p_{n} \leqslant p_{m}$ for all $n<m$. Then for any $n \in \mathbb{N}$ there are $m \in \mathbb{N}$ and $K_{n}>0$ so that

$$
\inf _{z \in Z^{1}(\Gamma, V)} p_{n}(c+z) \leqslant K_{n} q_{m}\left(\partial^{1}(c)\right)
$$

where $q_{m}$ is the continuous seminorm on $\operatorname{Hom}_{\Gamma}\left(R_{a b}, V\right)$ defined by

$$
q_{m}(h)=\max _{i \leqslant m} p_{m}\left(h\left(r_{i}\right)\right)
$$

for $h \in \operatorname{Hom}_{\Gamma}\left(R_{a b}, V\right)$.

Proof: By Theorem 2.5, $B^{2}(\Gamma, V)$ is a closed subspace of $Z^{2}(\Gamma, V)$ and so is a Fréchet space and so $\partial^{1}: C^{1}(\Gamma, V) \rightarrow B^{2}(\Gamma, V)$ is a continuous, surjective linear map of Fréchet spaces. By [2, Proposition IV 4.1.1], given two Fréchet spaces $X$ and $Y$, a continuous linear mapping $L: X \rightarrow Y$ is a surjective linear mapping of Fréchet spaces if and only if for every continuous seminorm $p$ on $X$ there is a continuous seminorm $q$ on $Y$ with $\inf _{x^{\prime} \in \operatorname{ker}(L)} p\left(x+x^{\prime}\right) \leq(L . x)$ for all $x \in X$. Applying this to $X=C^{1}(\Gamma, V)$, $Y=B^{2}(\Gamma, V)$ and $L=\partial^{1}$, we find that for every continuous seminorm $p$ on $C^{1}(\Gamma, V)$ there is a seminorm $\widehat{q}$ on $B^{2}(\Gamma, V)$ with

$$
\inf _{z \in Z^{1}(\Gamma, V)} p(c+z) \leqslant q\left(\partial^{1}(c)\right)
$$

for all $c \in C^{1}(\Gamma, V)$. Note that the collection of seminorms $\left\{q_{m}\right\}_{m \in \mathbb{N}}$ form a fundamental system of seminorms for the topology on $\operatorname{Hom}_{\Gamma}\left(R_{a b}, V\right)$ and that for $m_{1} \leqslant m_{2}$, $q_{m_{1}} \leqslant q_{m_{2}}$. Therefore, for all $n$ there is a seminorm $\bar{q}_{n}$ on $\operatorname{Hom}_{\Gamma}\left(R_{a b}, V\right)$ so that

$$
\inf _{z \in Z^{1}(\Gamma, V)} p_{n}(c+z) \leqslant \bar{q}_{n}\left(\partial^{1} \cdot c\right)
$$

Put $\bar{U}_{n}=\left\{h \in \operatorname{Hom} \Gamma\left(R_{a b}, V\right) \mid \bar{q}_{n}(h)<1\right\}$ and $U_{m}=\left\{h \in \operatorname{Hom}_{\Gamma}\left(R_{a b}, V\right) \mid q_{m}(h)<\right.$ $1\}$. By definition, the sets $\lambda U_{m}$ for $\lambda>0$ form a fundamental system of neighbourhoods of 0. Consequently, $\bar{U}_{n}$ contains a neighbourhood $U$ of 0 of the form $U=\bigcap_{i=1, \ldots, N} \lambda U_{m_{i}}$. Put $\bar{\lambda}=\min \left\{\lambda_{m_{1}}, \ldots, \lambda_{m_{N}}\right\}$ and $m=\max \left\{m_{1}, \ldots, m_{N}\right\}$. Therefore $\bar{\lambda} U_{m} \subset U$ and so $U_{m} \subset(1 / \bar{\lambda}) U \subset(1 / \bar{\lambda}) \bar{U}_{n}$ and so $\bar{q}_{n}(h)=\inf \left\{\lambda \geqslant 0 \mid h \in \lambda \bar{U}_{n}\right\} \leqslant(1 / \bar{\lambda}) q_{m}(h)$ for all $h \in \operatorname{Hom}_{\Gamma}\left(R_{a b}, V\right)$. This provides the conclusion of the proof with $K_{n}=(1 / \lambda)$. $]$

Theorem 2.7 forms the basis for approximating cochains by cocycles. The idea is that a cochain whose image under the coboundary map is small, must have a small seminorm in an appropriate seminorm on the quotient of the cochains by the coboundaries. 
Combining this estimate with the (not necessarily linear) section of the coboundary map produces a cocycle approximating the original cochain. The following Corollary makes this idea precise for the case that $V$ is a Banach space.

COROLlary 2.8. With the hypotheses of Theorem 2.7 and the additional assumption that $V$ is a Banach space with norm \|\| , there is $n \in \mathbb{N}$ and $K>0$ so that for any $c \in C^{1}(\Gamma, V)$ there is $z \in Z^{1}(\Gamma, V)$ satisfying

$$
\|c-z\| \leqslant K \min _{1 \leqslant i \leqslant n}\left\|\left(\partial^{1} c\right)\left(r_{i}\right)\right\| .
$$

Proof: By Theorem 2.7 there are $m \in \mathbb{N}$ and $K_{m}>0$ so that

$$
\inf _{z \in Z^{1}(\Gamma, V)}\|c+z\| \leqslant K_{m} \min _{1 \leqslant i \leqslant m}\left\|\left(\partial^{1} c\right)\left(r_{i}\right)\right\| .
$$

By Proposition 2.2, there are also a constant $L>0$ and a section $\tau: C^{1} / Z^{1} \rightarrow C^{1}$ satisfying

$$
\left\|\tau\left(c+Z^{\mathbf{1}}(\Gamma, V)\right)\right\| \leqslant L \inf _{z \in Z^{1}(\Gamma, V)}\|c+z\| .
$$

By applying the previous Theorem, setting $p_{n}=p_{m}=\|\|$ (since $V$ is a Banach space), $\inf _{z \in Z^{1}(\Gamma, V)}\|c+z\| \leqslant K_{n} \min _{1 \leqslant i \leqslant n}\left\|\partial^{1}(c)\left(r_{i}\right)\right\|$. Hence,

$$
\left\|c-\left(c-\tau\left(c+Z^{1}(\Gamma, V)\right)\right)\right\| \leqslant L K_{n} \min _{1 \leqslant i \leqslant n}\left\|\partial^{1}(c)\left(r_{i}\right)\right\|,
$$

completing the proof with $K=L K_{n}$ and $z=c-\tau\left(c+Z^{1}(\Gamma, V)\right)$.

\section{STABILITY}

PRELIMINARIES. Several preliminary results needed for the proof of the main theorem will be collected here. Some of these are fairly simple and are folklore; for these the proofs will be omitted or only briefly indicated. There are one or two of these results for which full proofs will be given however. Fix a presentation $\Gamma=\left\langle\gamma_{1}, \ldots, \gamma_{n} \mid r_{1}, \ldots\right\rangle$ with the corresponding short exact sequence of groups $1 \longrightarrow R \rightarrow F \rightarrow \Gamma \longrightarrow 1$ where $F$ is freely generated by $\gamma_{1}, \ldots, \gamma_{n}$ and $R$ is freely generated (by the NielsenSchrier Theorem, [12, Theorem 6.1.1]) by $r_{1}, \ldots$ For the remainder of the section, this will be fixed.

The first result is a routine application of the identity

$$
F(y)=F(x)+\int_{0}^{1} d F(x+\lambda(y-x)) \cdot(y-x) d \lambda
$$

for any $C^{1}$ mapping $F: X \rightarrow Y$ of a Banach space $X$ to a Banach space $Y$. 
Lemma 3.1. Let $F$ be a $C^{1}$ function from a Banach space $X$ to a Banach space $Y$ and $U \subset X$ a closed, bounded, convex subset of $X$. If $A \in C L(X, Y)$ satisfies $\|A x-d F(u) . x\|_{Y} \leqslant \varepsilon\|x\|_{X}$ for all $x \in X$ and $u \in U$ then $\| F(y)-F(x)-$ $A(y-x)\left\|_{Y} \leqslant \varepsilon\right\| y-x \|_{X}$ for all $x, y \in U$.

The next results apply the results of the previous section to constructing the necessary estimates for approximating a $C^{1} \Gamma$ action by cocycles in the neighbourhood of a fixed point of a fixed $\Gamma$ action.

Proposition 3.2. Let $V$ a Banach space, $\Gamma$ a finitely generated group, with presentation as above, $\phi \in \operatorname{Act}^{1}(\Gamma, V)$ an action that fixes 0 and $\Phi \in R(\Gamma, G L(v))$ the action of $\Gamma$ on $V$ obtained by linearising $\phi$ at 0 . Let $F_{n} \subset F$ be the subset of $F$ consisting of reduced words in $F$ whose reduced length is less than $n \in \mathbb{N}$. For any $n \in \mathbb{N}$ there are constants $L_{n}$ and $K_{n}>0$ depending only on $n$, so that for any convex, bounded neighbourhood $U \subset V$ of 0 there are neighbourhoods $W \subset \operatorname{Act}^{1}(\Gamma, V)$ of $\phi$ and convex $U^{\prime} \subset U$ of 0 so that for any neighbourhoods $W^{\prime} \subset W$ and convex $\widehat{U} \subset U^{\prime}$ of $\phi$ and 0 respectively of the form

$$
\begin{aligned}
& W^{\prime}=\left\{\psi \in W \mid\|\psi(\gamma)(x)-\phi(\gamma)(x)\| \leqslant \varepsilon_{0}\right. \text { and } \\
& \left.\quad\|d \psi(\gamma)(x) \cdot v-d \phi(\gamma)(x) \cdot v\| \leqslant \varepsilon_{1}\|v\| \text { for all } x \in U, v \in V \text { and } \gamma \in S\right\} \\
& \widehat{U} \subset\left\{x \in U^{\prime} \mid\|d \phi(\gamma)(x) \cdot v-d \phi(\gamma)(0) . v\| \leqslant \varepsilon_{1}\|v\| \text { for all } v \in V \text { and } \gamma \in S\right\}
\end{aligned}
$$

where $\varepsilon_{0}, \varepsilon_{1}>0$, such that for all $x, y \in \widehat{U}$,

$$
\left\|\psi(w)(x)-\Phi(w) .(x-y)-y-d w_{\Phi}\left(\psi_{y}\right)\right\| \leqslant \varepsilon_{1}\left(L_{n}\|x-y\|+K_{n}\left\|\psi_{y}\right\|\right)
$$

where $\psi_{y}$ is the element of $C^{1}(\Gamma, V)$ defined by $\psi_{y}\left(\gamma_{i}\right)=\psi\left(\gamma_{i}\right)(y)-y$ for $i=1, \ldots, n$.

Proof: Let $E: V \times \operatorname{Act}^{1}(\Gamma, V) \rightarrow V^{\left|F_{n}\right|}$ be the function

$$
E(x, \psi)_{w}=\psi\left(w\left(\gamma_{1}, \ldots, \gamma_{n}\right)\right)(x) \text { for all } w \in F_{n}
$$

Continuity of the evaluation map ev: $V \times \operatorname{Act}^{1}(\Gamma, V) \rightarrow V$ and the hypothesis that $\phi(\gamma)(0)=0 \in U$ for all $\gamma \in \Gamma$ imply that there are neighbourhoods $W$ of $\phi$ in $\operatorname{Act}^{1}(\Gamma, V)$ and $U_{n}$ of 0 in $V$ so that $E(x, \psi) \in U^{\left|F_{n}\right|}$ for all $(x, \psi) \in U_{n} \times W$. Since the trivial word $w=1 \in F_{n}$ for all $n \in \mathbb{N}$ it is clear that $U_{n} \subset U$. Moreover, $U_{n}$ may be chosen so that $U_{n} \subset \widehat{U}_{n-1}$ and is convex for all $n>1$.

The neighbourhoods $W$ and $U_{n}$ are the candidates for the $W$ and $U^{\prime}$ of the Proposition. 
Let $A_{n}=\max _{w \in F_{n}}\left\|\Phi\left(w\left(\gamma_{1}, \ldots, \gamma_{n}\right)\right)\right\|$ and $B_{n}=\max _{w \in F_{n}}\left\|d w_{\Phi}\right\|$. Note that $A_{n} \leqslant A_{1}^{n}$ and $B_{n} \leqslant 1+A_{1} B_{n-1}$. Without loss of generality, assume that $\varepsilon_{1}<1 / 2$. It is easily verified that by Lemma 3.1

$$
\left\|\psi(\gamma)(x)-\left[\Phi(\gamma) \cdot(x-y)+y+d \gamma_{\Phi}\left(\psi_{y}\right)\right]\right\| \leqslant 2 \varepsilon_{1}\|x-y\|
$$

for all $x, y \in \widehat{U}$ and $\gamma \in S$.

Since $\psi(\gamma)\left(\psi\left(\gamma^{-1}\right)(x)\right)=x$ for all $x \in V$, for all $x \in \widehat{U}$, substituting $\psi\left(\gamma^{-1}\right)(x)$ for $x$ in the above and rearranging gives

$$
\left\|_{1} x-y-\Phi(\gamma) \cdot\left(\psi\left(\gamma^{-1}\right)(x)-y\right)-d \gamma_{\Phi}\left(\psi_{y}\right)\right\| \leqslant 2 \varepsilon_{1}\left\|\psi\left(\gamma^{-1}\right)(x)-y\right\|
$$

for all $x, y \in \widehat{U}$ and $\gamma \in S$. Since $d \gamma_{\Phi}^{-1}\left(\psi_{y}\right)=-\Phi\left(\gamma^{-1}\right) \cdot d \gamma_{\Phi}\left(\psi_{y}\right)$, factoring out a common factor of $\Phi\left(\gamma^{-1}\right)$ from $\psi\left(\gamma^{-1}\right)(x)-\left[\Phi\left(\gamma^{-1}\right) \cdot(x-y)+y+d \gamma_{\Phi}^{-1}\left(\psi_{y}\right)\right]$ gives

$$
\begin{aligned}
& \left\|\psi\left(\gamma^{-1}\right)(x)-\left[\Phi\left(\gamma^{-1}\right) \cdot(x-y)+y+d \gamma_{\Phi}^{-1}\left(\psi_{y}\right)\right]\right\| \\
& \quad=\left\|\Phi\left(\gamma^{-1}\right) \cdot\left[\Phi(\gamma) \cdot\left(\psi\left(\gamma^{-1}\right)(x)-y\right)-(x-y)+d \gamma_{\Phi}\left(\psi_{y}\right)\right]\right\| \\
& \quad \leqslant 2 \varepsilon_{1}\left\|\Phi\left(\gamma^{-1}\right)\right\|\left\|\psi\left(\gamma^{-1}\right)(x)-y\right\| \\
& \quad \leqslant 2 \varepsilon_{1} A_{1}\left(\left\|\psi\left(\gamma^{-1}\right)(x)-\psi\left(\gamma^{-1}\right)(y)\right\|+\left\|\psi_{y}\right\|\right)
\end{aligned}
$$

for all $x, y \in \widehat{U}$ and $\gamma \in S$. Moreover, by Proposition 3.1

$$
\left\|\psi\left(\gamma^{-1}\right)(x)-\psi\left(\gamma^{-1}\right)(y)\right\| \leqslant\left(A_{1}+2 \varepsilon_{1}\right)\|x-y\|
$$

and so

$$
\left\|\psi\left(\gamma^{-1}\right)(x)-\Phi\left(\gamma^{-1}\right) \cdot(x-y)-y-d \gamma_{\Phi}^{-1}\left(\psi_{y}\right)\right\| \leqslant \varepsilon_{1}\left(\left(2 A_{1}^{2}+A_{1}\right)\|x-y\|+2 A_{1}\left\|\psi_{y}\right\|\right)
$$

for all $x, y \in \widehat{U}$ and $\gamma \in S$. By putting $K_{1}=2 A_{1}^{2}+A_{1}$ and $L_{1}=2 A_{1}$ the result is true for $\mathrm{n}=1$. The remainder of the proof is by induction on $n$. Assume that the result is true for $F_{k}$ where $k<n$. Let $w \in F_{n}-F_{n-1}$. Then there are words $w_{1}$ and $w_{2}$ in $F_{n-1}$ so that $w=w_{1} w_{2}$ hence, after some manipulation,

$$
\begin{aligned}
&\left\|\psi\left(w_{1} w_{2}\right)(x)-\Phi\left(w_{1} w_{2}\right) \cdot(x-y)-y-d\left(w_{1} w_{2}\right)_{\Phi}\left(\psi_{y}\right)\right\| \\
& \leqslant\left\|\psi\left(w_{1}\right)\left(\psi\left(w_{2}\right)(x)\right)-\Phi\left(w_{1}\right) \cdot\left(\psi\left(w_{2}\right)(x)-y\right)-y-d w_{1 \Phi}\left(\psi_{y}\right)\right\| \\
&+\left\|\Phi\left(w_{1}\right) \cdot\left(\psi\left(w_{2}\right)(x)-y\right)-\Phi\left(w_{1}\right) \cdot \Phi\left(w_{2}\right) \cdot(x-y)-\Phi\left(w_{1}\right) \cdot d w_{2 \Phi}\left(\psi_{y}\right)\right\| \\
& \leqslant \varepsilon_{1} L_{n-1}\left\|\psi\left(w_{2}\right)-y\right\|+\varepsilon_{1} K_{n-1}\left\|\psi_{y}\right\| \\
&+\left\|\Phi\left(w_{1}\right)\right\| \varepsilon_{1}\left(L_{n-1}\|x-y\|+K_{n-1}\left\|\psi_{y}\right\|\right) .
\end{aligned}
$$


Since

$$
\begin{aligned}
\mid\left\|\psi\left(w_{2}\right)(x)-y\right\|-\| \Phi\left(w_{2}\right) \cdot(x-y) & +d w_{2 \Phi}\left(\psi_{y}\right) \| \mid \\
& \leqslant\left\|\psi\left(w_{2}\right)(x)-\Phi\left(w_{2}\right) \cdot(x-y)-y-d w_{2 \Phi}\left(\psi_{y}\right)\right\|
\end{aligned}
$$

by the inductive hypothesis,

$$
\left|\left\|\psi\left(w_{2}\right)(x)-y\right\|-\left\|\Phi\left(w_{2}\right) \cdot(x-y)+d w_{2 \Phi}\left(\psi_{y}\right)\right\|\right| \leqslant \varepsilon_{1}\left(L_{n-1}\|x-y\|+K_{n-1}\left\|\psi_{y}\right\|\right),
$$

and so on rearranging terms

$$
\begin{aligned}
\left\|\psi\left(w_{2}\right)(x)-y\right\| \leqslant & \left\|\Phi\left(w_{2}\right) \cdot(x-y)+d w_{2 \Phi}\left(\psi_{y}\right)\right\| \\
& \quad+\varepsilon_{1}\left(L_{n-1}\|x-y\|+K_{n-1}\left\|\psi_{y}\right\|\right) \\
\leqslant & \left(A_{n-1}+\varepsilon_{1} L_{n-1}\right)\|x-y\|+\left(B_{n-1}+\varepsilon_{1} K_{n-1}\right)\left\|\psi_{y}\right\|
\end{aligned}
$$

Substituting, the result now follows with constants $L_{n}$ and $K_{n}$ given by the formulae

$$
\begin{aligned}
L_{n} & =A_{n-1} L_{n-1}+\frac{1}{2} L_{n-1}^{2}+B_{n-1} \quad \text { and } \\
K_{n} & =B_{n-1}+K_{n-1} A_{n-1}+\frac{1}{2} L_{n-1} K_{n-1}
\end{aligned}
$$

since $\varepsilon_{1}$ was chosen less than $1 / 2$.

Proposition 3.3. Let $V$ a Banach space, $\Gamma$ finitely generated, $\phi \in \operatorname{Act}^{1}(\Gamma, V)$ an action of $\Gamma$ on $V$ fixing $0, \Phi \in R(\Gamma, G L(v))$ the linearisation of $\phi$ at 0 with $B^{2}(\Gamma, V)$ a closed subspace of $Z^{2}(\Gamma, V)$. There is a $K>0$ so that for any convex, bounded neighbourhood $U \subset V$ of 0 , there are neighbourhoods $U_{1} \subset U$ of 0 and $W \subset \operatorname{Act}^{1}(\Gamma, V)$ of $\phi$ so that for all neighbourhoods $W^{\prime} \subset W$ and convex neighbourhoods $U^{\prime} \subset U_{1}$ of $\phi$ and 0 respectively of the form

$$
\begin{aligned}
& W^{\prime}=\left\{\psi \in W \mid\|\psi(\gamma)(x)-\phi(\gamma)(x)\| \leqslant \varepsilon_{0}\right. \text { and } \\
& \left.\qquad d \psi(\gamma)(x) . v-d \phi(\gamma)(x) . v\left\|\leqslant \varepsilon_{1}\right\| v \| \text { for all } x \in U, v \in V \text { and } \gamma \in S\right\}
\end{aligned}
$$

and

$$
U^{\prime} \subset\left\{x \in U_{1} \mid\|d \phi(\gamma)(x) . v-\Phi(\gamma) . v\| \leqslant \varepsilon_{1}\|v\| \text { for all } x \in U, v \in V \text { and } \gamma \in S\right\}
$$

where $\varepsilon_{0}$ and $\varepsilon_{1}$ are greater than zero, for any $\psi \in W^{\prime}$ and $y \in U_{1}$ the 1-cochain $\psi_{y}$ defined by $\psi_{y}\left(\gamma_{i}\right)=\psi\left(\gamma_{i}\right)(y)-y$ satisfies

$$
\inf _{z \in Z^{1}(\Gamma, V)}\left\|\psi_{y}+z\right\| \leqslant K \varepsilon_{1}\left\|\psi_{y}\right\|
$$


where $\|c\|=\max _{i=1, \ldots, n}\left\{\left\|c\left(\gamma_{i}\right)\right\|\right\}$ for all $c \in C^{1}(\Gamma, V)$.

Proof: By Corollary 2.8, there is an $n>0$ so that

$$
\inf _{z \in Z^{1}(\Gamma, V)}\left\|\psi_{y}+z\right\| \leqslant\left\|\delta^{1} \cdot \psi_{y}\right\|_{n}
$$

where $\|h\|_{n}=\max _{i=1, \ldots, n}\left\|h\left(r_{i}\right)\right\|$. Therefore, by definition,

$$
\inf _{z \in Z^{1}(\Gamma, V)}\left\|\psi_{y}+z\right\| \leqslant \max _{i=1, \ldots, n}\left\|d r_{i \Phi} \psi_{y}\right\| .
$$

Let $l_{i}=\operatorname{length}\left(r_{i}\right)$ for $i=1, \ldots, n$ be the length of the (reduced) word $r_{i}$ and $N=\max \left\{l_{i}\right\}$. By Proposition 3.2, for this value of $N$ and all neighbourhoods $U$ of 0

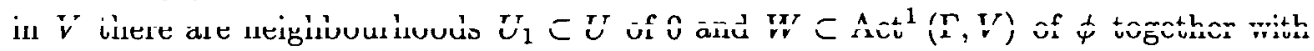
$L_{N}>0$ and $K_{N}>0$ so that for any neighbourhoods $W^{\prime} \subset W$ and $U^{\prime} \subset U_{1}$ of $\phi$ and 0 of the form

$$
\begin{aligned}
& W^{\prime}=\left\{\psi \in \operatorname{Act}^{1}(\Gamma, V) \mid\|\psi(\gamma)(x)-\phi(\gamma)(x)\| \leqslant \varepsilon_{0}\right. \text { and } \\
& \left.\quad\|d \psi(\gamma)(x) \cdot v-d \phi(\gamma)(x) . v\| \leqslant \varepsilon_{1}\|v\| \text { for all } x \in U, v \in V \text { and } \gamma \in S\right\}
\end{aligned}
$$

and

$$
U^{\prime} \subset\left\{x \in U_{1} \mid\|d \phi(\gamma)(x) . v-\Phi(\gamma) \cdot v\| \leqslant \varepsilon_{1}\|v\| \text { for all } x \in U, v \in V \text { and } \gamma \in S\right\}
$$

where $\varepsilon_{0}, \varepsilon_{1}>0$ so that

$$
\left\|\psi\left(r_{j}\right)(x)-\Phi\left(r_{j}\right) \cdot(x-y)-y-d r_{j \Phi}\left(\psi_{y}\right)\right\| \leqslant \varepsilon_{1}\left(L_{N}\|x-y\|+K_{N}\left\|\psi_{y}\right\|\right)
$$

for all $x, y$ in $U^{\prime}$. Putting $x=y$ and observing that $\psi\left(r_{j}\right)(x)=\Phi\left(r_{j}\right) . x=x$ gives

$$
\left\|d r_{j \Phi}\left(\psi_{y}\right)\right\| \leqslant \varepsilon_{1} K_{N}\left\|\psi_{y}\right\|
$$

and so $\left\|\delta^{1}\left(\psi_{y}\right)\right\|_{n} \leqslant \varepsilon_{1} K_{N}\left\|\psi_{y}\right\|$. The result now follows with $K=k K_{N}$.

This completes the preliminary results necessary for the presentation of the stability theorem. The proof follows in the next section.

Main Theorem. The proof is constructive. Propositions 2.1 and 2.2 imply there are sections $\Delta^{0}$ and $\tau^{1}$ of the coboundary operators $\delta^{0}$ and $\delta^{1}$ respectively satisfying $\left\|\Delta^{i}(b)\right\| \leqslant L_{i}\|b\|$ for all $b \in B^{i}(\Gamma, V)$ for $i=0,1$. Estimates on the size of $L_{0}$ and $L_{1}$ give an a priori bound on $\varepsilon_{1}$ and $\varepsilon_{0}$ allowing Propositions 3.2 and 3.3 to construct (via $\Delta^{1}$ ) a sequence of cocycles which are then converted into approximate fixed points using $\Delta^{0}$. The bounds derived for $\varepsilon_{0}$ and $\varepsilon_{1}$ ensure that the elements of this sequence remain in the neighbourhood of the original fixed point where Propositions 3.2 and 3.3 are applicable. Finally, the sequence is shown to converge to a fixed point. The result is initially proved for actions on a Banach space and the generalisation to more general Banach manifolds is shown to follow from this case. 
ThEOREM 3.4. Let $\Gamma$ be a finitely generated group, $M$ a $C^{2}$ Banach manifold modelled on a Banach space $V, \phi \in \operatorname{Act}^{1}(\Gamma, M)$ an action of $\Gamma$ on $M$ satisfying

(1) $\phi$ fixes a point $p \in M$

(2) if $\Phi \in R(\Gamma, G L(V))$ is the linear action obtained by linearisation of $\phi$ at $p$, then $H^{1}(\Gamma, V)=0$, where $V$ is a $\Gamma$ module via $\Phi$.

Then, $p$ is a stable fixed point, that is, for all neighbourhoods $U$ of $p$ there is a neighbourhood $W$ of $\phi$ in $\operatorname{Act}^{1}(\Gamma, V)$ so that all actions $\psi \in W$ have fixed points in $U$.

PROOF: The proof will be carried out for the case $M=V$. The general case will be shown to reduce to this. By hypothesis, the images of the coboundary maps $\delta^{0}$ and $\delta^{1}$ are Fréchet spaces. By Proposition 2.2 there is a section $\tau^{1}$, of the quotient map $\pi^{1}: C^{1}(\Gamma, V) \rightarrow C^{1}(\Gamma, V) / Z^{1}(\Gamma, V)$ satisfying $\left\|\tau^{1}(\widehat{c})\right\| \leqslant \widehat{K}\|\widehat{c}\|$ for some $\widehat{K}>0$ and all $\widehat{c} \in C^{1}(\Gamma, V) / Z^{1}(\Gamma, V)$. Define $D_{1}>0$ by $D_{1}=\widehat{K} K$ where $K$ is the constant whose existence is guaranteed by Proposition 3.3. Similarly, by Proposition 2.2 there is a continuous section $\Delta^{0}: B^{1}(\Gamma, V) \rightarrow V$ of the 0 -th coboundary operator that satisfies $\left\|\Delta^{0}(b)\right\| \leqslant D_{0}\|b\|$ for all $b \in B^{1}(\Gamma, V)$. Let $d_{0}>0$ be a positive real number so that $\left\|\delta^{0}(v)\right\| \leqslant d_{0}\|v\|$ for all $v \in V$ and $m \in \mathbb{N}$ so that $m \geqslant D_{1}$. Define constants $\eta_{0}$ and $\eta_{1}$ by

$$
\eta_{1}=D_{1}+D_{0}\left(1+1 / m D_{1}\right) \quad \text { and } \quad \eta_{0}=\frac{1+1 / m D_{1}}{1-1 / m D_{1}} \eta_{1}
$$

These choices are not optimal, just workable.

The next step is to construct a priori bounds on the size of neighbourhoods of $\phi$ in $\operatorname{Act}^{1}(\Gamma, M)$ so that all actions in the constructed neighbourhoods satisfy the conclusion of the theorem. To do so, choose an $\varepsilon_{1}>0$ so that

$$
\varepsilon_{1} \ll \min \left\{\frac{1}{m}, \frac{1}{\eta_{0}}\right\}
$$

This will remain fixed throughout the remainder of the proof. By Proposition 3.3, there are a neighbourhoods $U^{\prime} \subset U$ and $W \subset \operatorname{Act}^{1}(\Gamma, V)$ of $\phi$ respectively, so that

$$
\inf _{z \in Z^{1}(\Gamma, V)}\left\|\psi_{x}+z\right\| \leqslant K \varepsilon_{1}\left\|\psi_{x}\right\|
$$

for all $x \in \widehat{U}$ and $\psi \in W^{\prime}$ that are neighbourhoods of 0 and $\phi$ respectively of the form

$$
\begin{aligned}
\widehat{U} \subset\left\{x \in U^{\prime} \mid\|d \phi(\gamma)(x) \cdot v-d \phi(\gamma)(0) . v\| \leqslant \varepsilon_{1}\|v\| \text { for all } v \in V \text { and } \gamma \in S\right\} & \\
W^{\prime}= & \left\{\psi \in W \mid\|\psi(\gamma)(x)-\phi(\gamma)(x)\| \leqslant \varepsilon_{0}^{\prime}\right. \text { and } \\
& \left.\|d \psi(\gamma)(x) \cdot v-d \phi(\gamma)(x) \cdot v\| \leqslant \varepsilon_{1}^{\prime}\|v\| \text { for all } x \in U, v \in V \text { and } \gamma \in S\right\}
\end{aligned}
$$


respectively and $\psi_{x} \in C^{1}(\Gamma, V)$ is the 1-cochain defined by $\psi_{x \gamma}=x-\psi(\gamma)(x)$ for $\gamma \in S$. Without loss of generality it can be assumed that $\widehat{U}$ is convex. Let $W_{U 1} \subset \operatorname{Act}^{1}(\Gamma, V)$ be the neighbourhood of $\phi$ defined by

$$
W_{U, 1}=\left\{\psi \in W \mid\|d \psi(\gamma)(x) . v-d \phi(\gamma)(x) . v\| \leqslant \varepsilon_{1}\|v\| \text { for all } x \in U, v \in V, \gamma \in S\right\} .
$$

Given such a neighbourhood $U$ of 0 , choose $r>0$ small enough that $\{x \in V \mid\|x\| \leqslant$ $r\} \subset \widehat{U}$. Choose $\varepsilon_{0}>0$ so that

$$
\varepsilon_{0} D_{0} \frac{\varepsilon_{1} \eta_{0}}{\left(1-\varepsilon_{1} \eta_{0}\right)\left(1-1 / m D_{1}\right)} \ll r
$$

Now let $W_{U, 0}$ be the neighbourhood of $\phi$ defined by

$$
W_{U, 0}=\left\{\psi \in W_{U, 1} \mid\|\psi(\gamma)(x)-\phi(\gamma)(x)\| \leqslant \varepsilon_{0} \text { for all } x \in U \text { and } \gamma \in S\right\} .
$$

The remainder of the proof will show that given $U$ as above, actions $\psi$ in $W_{U, 0}$ have fixed points in $\{x \in V \mid\|x\| \leqslant r\}$ where $r$ is as above.

Given $\psi \in W^{\prime}$, the strategy will be to construct a sequence of approximate cocycles $\psi_{j} \in C^{1}(\Gamma, V)$ which measure the failure of the $j$ th point to be a fixed point and to use the a priori estimates derived in Propositions 3.2 and 3.3 to construct true cocycles $z_{j} \in Z^{1}(\Gamma, V)$. It is then straightforward to construct approximate fixed points $x_{j}$ from the cocycles $z_{j}$. The a priori estimates are then shown to imply that $\left\|x_{j}\right\| \leqslant r$ for all $j$ and that the sequence of $x_{j}$ s converges to $x_{\infty}$ which is then shown to be a fixed point.

At the $j$ th step, define $\psi_{j} \in C^{1}(\Gamma, V), z_{j} \in Z^{1}(\Gamma, V)$ and $x_{j+1}$ by

$$
\begin{gathered}
\psi_{j \gamma}=x_{j}-\psi(\gamma)\left(x_{j}\right) \text { for } \gamma \in S \\
z_{j}=\psi_{j}-\tau^{1}\left(\pi\left(\psi_{j}\right)\right) \text { and } \\
x_{j+1}=x_{j}+\Delta^{0}\left(z_{j}\right)
\end{gathered}
$$

where $x_{0}=0$. Note that $\psi_{x}=0$ iff $x$ is a fixed point and since $\tau^{1}(0)=\Delta^{0}(0)=0$, a limit point of the sequence is a fixed point (assuming that the sequence converges within the ball $\|x\| \leqslant r)$. The proof proceeds by induction. The idea is to show for all $j \in \mathbb{N}$ that

$$
\left\|x_{j}\right\| \leqslant \varepsilon_{0} D_{0} \frac{1-\left(\varepsilon_{1} \eta_{0}\right)^{j}}{\left(1-\varepsilon_{1} \eta_{0}\right)\left(1-1 / m D_{1}\right)} \leqslant r .
$$

We begin the induction at $j=0$. By definition, $x_{0}=0 \in U^{\prime}$ and so by Proposition 3.3 ,

$$
\inf _{z \in Z^{1}(\Gamma, V)}\left\|\psi_{0}+z\right\| \leqslant \varepsilon_{1} K\left\|\psi_{0}\right\|
$$


Hence,

$$
\left\|z_{0}-\psi_{0}\right\| \leqslant\left\|\tau^{1}\left(\pi\left(\psi_{0}\right)\right)\right\| \leqslant \varepsilon_{1} D_{1}\left\|\psi_{0}\right\|
$$

Using the triangle inequality, this gives

$$
\left(1-1 / m D_{1}\right)\left\|\psi_{0}\right\| \leqslant\left\|z_{0}\right\| \leqslant\left(1+1 / m D_{1}\right)\left\|\psi_{0}\right\|
$$

and so after substituting into the inequality for $\left\|\psi_{0}-z_{0}\right\|$

$$
\left\|x_{1}\right\| \leqslant\left\|\Delta^{0}\left(z_{0}\right)\right\| \leqslant D_{0}\left(1+1 / m D_{1}\right) \varepsilon_{0} \leqslant r
$$

and so $\left\|x_{1}\right\| \leqslant r$.

By hypothesis, $\left\|x_{i}\right\| \leqslant r$ for $i<j$. By definition of $W_{U, 1}$, for all $x \in U$ and $v \in V$,

$$
\left\|d \psi_{j}(\gamma)(x) \cdot v-\Phi(\gamma) \cdot v\right\| \leqslant \varepsilon_{1}\|v\|
$$

Therefore by definition of $\delta^{0}$,

$$
\left\|v-d \psi(\gamma)(x) \cdot v-\delta^{0}(v)\right\| \leqslant \varepsilon_{1}\|v\| \quad \text { for all } \gamma \in S \text { and } x \in U
$$

so by Proposition 3.1

$$
\left\|\psi_{j}-\psi_{j-1}-\delta^{0}\left(x_{j}-x_{j-1}\right)\right\|=\varepsilon_{1}\left\|\Delta^{0}\left(z_{j-1}\right)\right\| \leqslant \varepsilon_{1} D_{0}\left\|z_{j-1}\right\|
$$

Expanding the left hand side of this inequality leads to the following bounds for $\left\|\psi_{j}-\psi_{j-1}\right\|:$

$$
\begin{aligned}
\left\|\psi_{j}-\psi_{j-1}-\delta^{0}\left(x_{j}-x_{j-1}\right)\right\| & \geqslant\left|\left\|\psi_{j}\right\|-\left\|\psi_{j-1}-\delta^{0}\left(x_{j}-x_{j-1}\right)\right\|\right| \\
& =\left|\left\|\psi_{j}\right\|-\left\|\psi_{j-1}-z_{j-1}\right\|\right|
\end{aligned}
$$

and so to the following bound for $\left\|\psi_{j}\right\|$ :

$$
\left\|\psi_{j}\right\| \leqslant\left\|\psi_{j-1}-z_{j-1}\right\|+\varepsilon_{1} D_{0}\left\|z_{j-1}\right\|
$$

By construction,

$$
\left\|z_{j-1}-\psi_{j-1}\right\| \leqslant\left\|\tau^{1}\left(\pi\left(\psi_{j-1}\right)\right)\right\| \leqslant \widehat{K} \inf _{z \in Z^{1}(\Gamma, V)}\left\|\psi_{j-1}+z\right\|
$$

and so by Proposition 3.3, this gives

$$
\left\|z_{j-1}-\psi_{j-1}\right\| \leqslant \varepsilon_{1} D_{1}\left\|\psi_{j-1}\right\|
$$


Again, by hypothesis, $\left\|x_{j}\right\|<r$ and so again by Proposition 3.3,

$$
\left\|\psi_{j}-z_{j}\right\| \leqslant\left\|\tau^{1}\left(\pi\left(\psi_{j}\right)\right)\right\| \leqslant \varepsilon_{1} D_{1}\left\|\psi_{j}\right\|
$$

Since $\varepsilon_{1} \leqslant 1 / m$ this gives the following bounds for $z_{j}$ and $\psi_{j}$ :

$$
\left(1-1 / m D_{1}\right)\left\|z_{j}\right\| \leqslant\left\|\psi_{j}\right\| \leqslant\left(1+1 / m D_{1}\right)\left\|z_{j}\right\|
$$

Combining this inequality with the previous inequality for $\left\|\psi_{j}\right\|$ provides the following inductive bound for $\left\|\psi_{j}\right\|$ :

$$
\begin{aligned}
\left\|\psi_{j}\right\| & \leqslant \varepsilon_{1} D_{0}\left\|z_{j-1}\right\|+\varepsilon_{1} D_{1}\left\|\psi_{j-1}\right\| \\
& \leqslant \varepsilon_{1}\left(D_{1}+D_{0}\left(1+1 / m D_{1}\right)\right)\left\|\psi_{j-1}\right\| \\
& =\varepsilon_{1} \eta_{1}\left\|\psi_{j-1}\right\| .
\end{aligned}
$$

Rewriting this so that the bound is now in terms of $\left\|z_{j}\right\|$ instead of $\left\|\psi_{j}\right\|$ gives

$$
\begin{aligned}
\left\|z_{j}\right\| & \leqslant \frac{1}{1-1 / m D_{1}}\left\|\psi_{j}\right\| \\
& \leqslant \varepsilon_{1} \frac{\eta_{1}}{1-1 / m D_{1}}\left\|\psi_{j-1}\right\| \\
& \leqslant \varepsilon_{1} \eta_{1} \frac{1+1 / m D_{1}}{1-1 / m D_{1}}\left\|z_{j-1}\right\| \\
& =\varepsilon_{1} \eta_{0}\left\|z_{j-1}\right\| .
\end{aligned}
$$

Hence, the preceeding shows that

$$
\begin{aligned}
& \left\|\psi_{j}\right\| \leqslant\left(\varepsilon_{1} \eta_{1}\right)^{j}\left\|\psi_{0}\right\| \leqslant\left(\varepsilon_{1} \eta_{1}\right)^{j} \varepsilon_{0} \\
& \left\|z_{j}\right\| \leqslant\left(\varepsilon_{1} \eta_{0}\right)^{j}\left\|z_{0}\right\| \leqslant\left(\varepsilon_{1} \eta_{0}\right)^{j} \frac{\varepsilon_{0}}{\left(1-1 / m D_{1}\right)} .
\end{aligned}
$$

The formula for $\left\|x_{j}\right\|$ follows since

$$
\begin{aligned}
\left\|x_{j+1}\right\| & \leqslant\left\|x_{j}\right\|+\left\|\Delta^{0}\left(z_{j}\right)\right\| \\
& \leqslant \varepsilon_{0} D_{0} \frac{1-\left(\varepsilon_{1} \eta_{0}\right)^{j}}{\left(1-\varepsilon_{1} \eta_{0}\right)\left(1-1 / m D_{1}\right)}+D_{0}\left\|z_{j}\right\| \\
& \leqslant \varepsilon_{0} D_{0} \frac{1-\left(\varepsilon_{1} \eta_{0}\right)^{j}}{\left(1-\varepsilon_{1} \eta_{0}\right)\left(1-1 / m D_{1}\right)}+D_{0}\left(\varepsilon_{1} \eta_{0}\right)^{j} \frac{\varepsilon_{0}}{\left(1-1 / m D_{1}\right)} \\
& =\varepsilon_{0} D_{0} \frac{1-\left(\varepsilon_{1} \eta_{0}\right)^{j+1}}{\left(1-\varepsilon_{1} \eta_{0}\right)\left(1-1 / m D_{1}\right)}
\end{aligned}
$$


and so $\left\|x_{j}\right\| \leqslant r$ for all $j \in \mathbb{N}$ and the sequence $\left\{x_{j}\right\}_{j=0}^{\infty}$ is Cauchy. Therefore there is $x_{*} \in B_{r}(0)$ so that $x_{j} \rightarrow x_{*}$ as $j \rightarrow \infty$. As $\psi_{j} \rightarrow \psi_{x_{*}}$ as $j \rightarrow \infty$ and $\psi_{j} \rightarrow 0$, it follows immediately that $x_{*}$ is a fixed point for $\psi$.

The general case follows by observing that the preceding argument as well as those of Propositions 3.2 and 3.3 are local and so apply to germs of $\Gamma$ actions. Therefore, by restricting the neighbourhood of the fixed point to be contained in a coordinate chart, the preceeding argument can be applied to the germ of the $\Gamma$ action obtained by restricting $\phi$ to this neighbourhood. This gives the general case.

The following Corollary could have been proven by an implicit function theorem argument, though this would not have shown the connection to the more general theory for actions of finitely generated groups.

Corollary 3.5. Let $M, V, \Gamma, \phi$ and $p$ be as in Theorem 3.4 and assume $\Gamma$ is finite. Then for each neighbourhood $U$ of $p$ in $M$ there is a neighbourhood $W$ of $\phi$ so that each $\psi \in W$ has a fixed point in $U$.

Proof: Since $H^{i}(\Gamma, V)=0$ for all $i>0$, the result follows.

\section{Applications}

The stability theorem of the previous chapter is applied to provide a partial verification of a conjecture of Zimmer [15]. This is to the effect that if $M$ is a smooth closed manifold, $\Gamma$ a finitely generated group acting smoothly on $M$ via an action $\phi \in \operatorname{Act}^{\infty}(\Gamma, M)$, and if $H^{1}\left(\Gamma, D^{\infty}(M)\right)=0$ then $\phi$ is locally rigid in $\operatorname{Act}^{\infty}(M)$.

Generalisations of Weil's Local Rigidity Theorem. Theorem 3.4 and its Corollaries may be used to generalise Weil's criterion for local rigidity of representations of a finitely generated group in a Lie group. This argument was first noticed by Fleming [7]. We reproduce the argument here for completeness. Define a topological group $G$ to be a $C^{r}$ almost Lie group (or almost Lie group for short) if $G$ is a $C^{r}$ Banach manifold with model Banach space $V$ as well as a topological group. Note that it is not assumed that multiplication and inversion are $C^{r}$ maps. Examples of such groups are $D i f f^{r}(M)$ for $1 \leqslant r<\infty$, where $M$ is a smooth, closed manifold. Of course, any Lie group $G$ is an almost Lie group as is $C^{r}(M, G)$ where again, $M$ is a smooth closed manifold and $G$ is any Lie group. A $C^{r}$ almost Lie group pair $(G, H)$ consists of $C^{r}$ almost Lie groups $G$ and $H$ together with a continuous group homomorphism $\iota: H \rightarrow G$ that is an injective immersion of $H$ into a subgroup of $G$ so that for each $h \in H$ the map $A d_{\iota(h)}: G \rightarrow G$ defined by $A d_{\iota(h)}(g)=\iota(h) g \iota\left(h^{-1}\right)$ is a $C^{1}$ map. An example of a pair $(G, H)$ is of course $\left(D_{i f f}^{r}(M), D_{i f f^{r+1}}(M)\right)$ for $M$ a smooth, closed manifold. Also $\left(C^{r}(M, G), C^{r+1}(M, G)\right)$ is an example of a pair. In both examples, the homomorphism $\iota$ is induced by the inclusion $C^{r+1} \hookrightarrow C^{r}$. The "canonical" example is 
$\left(D_{i f f}(M), D_{i f f}^{r+1}(M)\right)$. It is also apparent that if $G$ is an "honest" Lie group then $(G, G)$ is also an almost Lie group pair. Theorem 3.4 can be applied to prove a local rigidity result for representations of a finitely generated group $\Gamma$ as follows.

THEOREM 4.1. Let $\Gamma$ be a finitely generated group, $(G, H)$ an almost Lie group pair and $\phi \in R(\Gamma, H)$ a representation satisfying $H^{1}\left(\Gamma, T_{e} G\right)=0$. Then there is a neighbourhood $U$ of $\phi$ in $R(\Gamma, H)$ so that any $\psi \in U$ is conjugate to $\phi$ via an element of $G$. The action of $\Gamma$ on $T_{e} G$ is given by

$$
v \in T_{e} G \mapsto d A d_{\iota(\phi(\gamma))} e . v
$$

far sll $\gamma \in \Gamma$ and $\because \leq T_{c} \Omega$

Proof: Define $E D: R(\Gamma, H) \rightarrow \operatorname{Act}^{1}(\Gamma, G)$ by

$$
E D(\psi) \mapsto \Psi \in \operatorname{Act}^{1}(\Gamma, G) \text { where } \Psi(\gamma)(g)=\iota(\psi(\gamma)) g \iota\left(\phi\left(\gamma^{-1}\right)\right) .
$$

It is a matter of checking the definitions to see that $E D$ is a continuous function from $R(\Gamma, H)$ to $\operatorname{Act}^{1}(\Gamma, G)$. Moreover, $E D(\phi)$ has a fixed point at $e \in G$ with

$$
d(E D(\phi))(\gamma)(e) \cdot v=d A d_{\iota(\phi(\gamma))}(e) . v \text { for all } \gamma \in \Gamma \text { and } v \in T_{e} G .
$$

By hypothesis, $H^{1}\left(\Gamma, T_{\mathrm{e}} G\right)=0$ and so by Theorem 3.4 for each neighbourhood $U$ of $e$ in $G$ there is a neighbourhood $W$ of $\operatorname{Ad}_{\iota(\phi)}$ in $\operatorname{Act}^{1}(\Gamma, G)$ where each $\Psi \in W$ has a fixed point $g_{0} \in U$. By the continuity of $E D, \bar{W}=E D^{-1}(W)$ is an open subset of $R(\Gamma, H)$ and so for all $\psi \in \bar{W}$ there is $g_{0} \in G$ with

$$
g_{0}=\psi(\gamma) g_{0} \phi\left(\gamma^{-1}\right)
$$

for all $\gamma \in \Gamma$. This $g_{0}$ provides the required conjugacy.

This has the following Corollaries.

Corollary 4.2. Let $G$ be a Banach Lie group with $T_{e} G=g(G), \Gamma$ a finitely generated group and $\phi \in R(\Gamma, G)$ a representation of $\Gamma$ with $H^{1}(\Gamma, \mathfrak{g})=0$. Then $\phi$ is locally rigid in $R(\Gamma, V)$.

The following Corollary of Theorem 4.1 is a partial verification of Zimmer's conjecture that the conclusion of Weil's local rigidity theorem is true if the Lie group $G$ and its Lie algebra $g(G)$ are replaced by $D_{i f f^{\infty}}(M)$ and $D^{\infty}(M)$ respectively, where $M$ is a smooth closed manifold.

COROLlaRY 4.3. If $M$ is a smooth closed manifold and $\Gamma$ is a finitely generated group acting on $M$ via $\phi \in \operatorname{Act}^{r}(\Gamma, M)$ satisfying $H^{1}\left(\Gamma, D^{r-1}(M)\right)=0$ then there is 
a neighbourhood $U$ of $\phi$ in $\operatorname{Act}^{r}(\Gamma, M)$ so that every $\psi \in U$ is conjugate to $\phi$ via an element of Diff ${ }^{r-1}(M)$.

Proof: Since $\operatorname{Diff}^{r}(M)$ is an almost Banach Lie group and the action of $\operatorname{Diff}^{r}(M)$ on $\operatorname{Diff}^{r-1}(M)$ via conjugation is $C^{1}$, this reduces to an application of Theorem 4.1. [

This result generalises [ 7 , Theorem 1]. The hypotheses of the above are weaker in that $\Gamma$ is only required to be finitely generated rather than finitely presented and the vanishing of $H^{1}$ implies the closed range condition imposed by Fleming, by Theorem 2.4. Unlike Fleming's result, this reduces to Stowe's theorem in the finite dimensional case. The final result is an application of the above to the case of a finite group action. This parallels a result of Palais [11].

Corollary 4.4. With $M$ as above and $\Gamma$ a finite group, then any $\phi \in$ $\operatorname{Act}^{r}(\Gamma, M)$ is locally rigid.

Proof: This follows by combining Corollary 4.3 with the observation that $H^{1}(\Gamma, V)=$ 0 for a $\Gamma$ module $V$ possessing the structure of a real vector space.

\section{REFERENCES}

[1] R. Abraham, Lectures of Smale on differential topology, (mimeographed lecture notes) (Columbia University, New York, NY, 1961).

[2] N. Bourbaki, Topological vector spaces Chapter 1-5 (Springer-Verlag, Berlin, Heidelberg, New York, 1987).

[3] A. Borel and N. Wallach, Continuous cohomology, discrete subgroups and representations of reductive groups, Annals of Mathematics Studies (Princeton University Press, Princeton, NJ, 1980).

[4] K. Deimling, Non-linear functional analysis (Springer-Verlag, Berlin, Heidelberg, New York, 1985).

[5] J. Eells, 'On the geometry of function spaces', in Symposia de topologia algebraica (Universidad Nacional Autónoma de México, Mexico City, 1958).

[6] D.G. Ebin and J.E. Marsden, 'Groups of diffeomorphisms and the motion of incompressible fluid', Annals of Math. 92 (1970), 102-163.

[7] P. Fleming, 'Structural stability and group cohomology', Trans. Amer. Math. Soc. 275 (1983), 791-809.

[8] S. Lang, Differential and Riemannian manifolds, 3rd ed. (Springer-Verlag, Berlin, Heidelber, New York, 1995).

[9] Y. Matsushima and S. Murakami, 'On vector bundle valued harmonic forms and automorphic forms on symmetric Riemannian manifolds', Ann. of Math. 78 (1963), 365-416.

[10] H. Omori, 'On the group of diffeomorphisms on a compact manifold', Proc. Sympos. Pure Math. 15 (1970), 167-184.

[11] R. Palais, 'Equivalence of nearby differentiable actions of a compact group', Bull. Amer. Math. Soc. 67 (1961), 362-364. 
[12] D.J.R. Robinson, A course in the theory of groups (Springer-Verlag, Berlin, Heidelberg, New York, 1982).

[13] D. Stowe, 'The stability of the stationary set of a group action', Proc. Amer. Math. Soc. 79 (1980), 139.

[14] A. Weil, 'Remarks on the cohomology of groups', Ann. of Math. 80 (1964), 149.

[15] R. Zimmer, 'Actions of semisimple groups and discrete subgroups', in Proceedings of the International Congress of Mathematicians, (Berkeley CA, 1986) (American Mathematical Society, Providence R.I., 1987), pp. 1247-1258.

Department of Mathematics

Penn State Altoona

3000 Ivyside Park

Altoona PA 16601

United States of America

e-mail: vwb2@psu.edu 